\title{
Asymptomatic solitary metastasis to the stomach from breast cancer: A case report
}

\author{
YUKI KANEKO $^{1}$, YUMIKO KOI $^{1}$, KEIKO KAJITANI $^{1}$, MASAHIRO OHARA $^{1}$ and YUTAKA DAIMARU $^{2}$ \\ ${ }^{1}$ Department of Breast Surgery; ${ }^{2}$ Section of Pathological Research and Laboratory, \\ JA Hiroshima General Hospital, Hatsukaichi, Hiroshima 738-8503, Japan
}

Received March 30, 2019; Accepted September 1, 2020

DOI: $10.3892 /$ mco.2020.2145

\begin{abstract}
Distant metastases from breast cancer are frequently found in bones, lungs and the liver. Metastasis to the stomach is rare, and its clinical presentation remains unclear. The present report describes a case of isolated gastric metastasis from breast cancer identified by contrast-enhanced computed tomography (CT). A 45-year-old female patient underwent right mastectomy and axillary lymph node dissection after preoperative chemotherapy for right invasive lobular breast carcinoma T4bN2M0, stage IIIB. Postoperative radiotherapy and endocrine therapy with tamoxifen for 5 years were performed. CT for postoperative follow-up at 52 years old revealed thickening of the stomach wall. Although the patient was asymptomatic, erosive mucosa was observed on the gastric body during gastroscopy. The gastric lesion was immunohistochemically diagnosed as metastatic luminal disease from the breast cancer. Positron emission tomography/CT revealed no abnormal accumulation suggesting metastasis to other organs. Palbociclib and fulvestrant treatment were initiated for gastric metastasis. Invasive lobular breast carcinoma results in gastrointestinal metastasis, including the stomach, more frequently than invasive ductal breast carcinoma. However, most gastric metastases occur simultaneously with systemic metastases. Solitary metastasis to the stomach without symptoms as in this case has rarely been reported. The possibility of gastric metastasis should be considered among the differential diagnoses, even in the absence of symptoms, when gastrointestinal abnormalities are seen on $\mathrm{CT}$ in patients with a history of breast cancer.
\end{abstract}

Correspondence to: Dr Masahiro Ohara, Department of Breast Surgery, JA Hiroshima General Hospital, 1-3-3 Jigozen, Hatsukaichi, Hiroshima 738-8503, Japan

E-mail: oharamas@hotmail.com

Abbreviations: CT, computed tomography; ILC, invasive lobular carcinoma; ER, estrogen receptor; PR, progesterone receptor; GCDFP, gross cystic disease fluid protein

Key words: breast cancer, gastric metastasis, computed tomography

\section{Introduction}

Breast cancer is one of the most frequent cancers in women (1). Approximately $80-90 \%$ of invasive breast cancers are invasive ductal carcinomas, and the most common sites of breast cancer metastasis are the bones, lungs and liver $(2,3)$.

Invasive lobular carcinoma (ILC) of the breast is the second most common type of invasive breast cancer, representing $\leq 10 \%$ of all invasive breast cancers (4). ILC mostly metastasizes to the bones, lungs, and liver, but has been reported to be associated with higher rates of metastasis to specific sites, such as the gastrointestinal tract, genitourinary tract, peritoneum, retroperitoneum, and leptomeninges when compared to invasive ductal carcinoma (5-7).

The stomach is infrequently reported as a site of breast cancer metastasis, accounting for 0.1 to $3.5 \%(8,9)$. Metastasis to the stomach generally follows disease extension, and solitary gastric metastasis is thus relatively rare (10). Due to this rarity, breast cancer with gastric metastasis is occasionally confused with primary cancer in the stomach. In addition, patient symptoms due to gastric metastasis such as indigestion, anorexia, and nausea are non-specific, and may be explained by other metastases or side effects of treatments such as chemotherapy (10). Gastric metastases are thus often overlooked when encountering gastrointestinal symptoms in patients.

The present report describes a case of isolated metastasis to the stomach from ILC as a first presentation of metastasis, identified from computed tomography (CT) in the absence of symptoms.

\section{Case report}

A 45-year-old woman underwent mastectomy with axillary lymph node dissection after preoperative chemotherapy (four courses of cyclophosphamide, epirubicin and 5-fluorouracil, followed by four courses of docetaxel) for an estrogen receptor (ER)-positive, HER2-negative invasive lobular cancer, T4bN2M0 stage IIIB (11). Postoperative radiation therapy with $50 \mathrm{~Gy}$ in 25 fractions to supraclavicular lymph nodes and chest wall was performed and 5 years endocrine therapy with tamoxifen $20 \mathrm{mg}$ once daily was completed. At 52 years old, CT performed as postoperative follow-up indicated thickening of the stomach wall with contrast enhancement (Fig. 1). Although the patient was asymptomatic, 


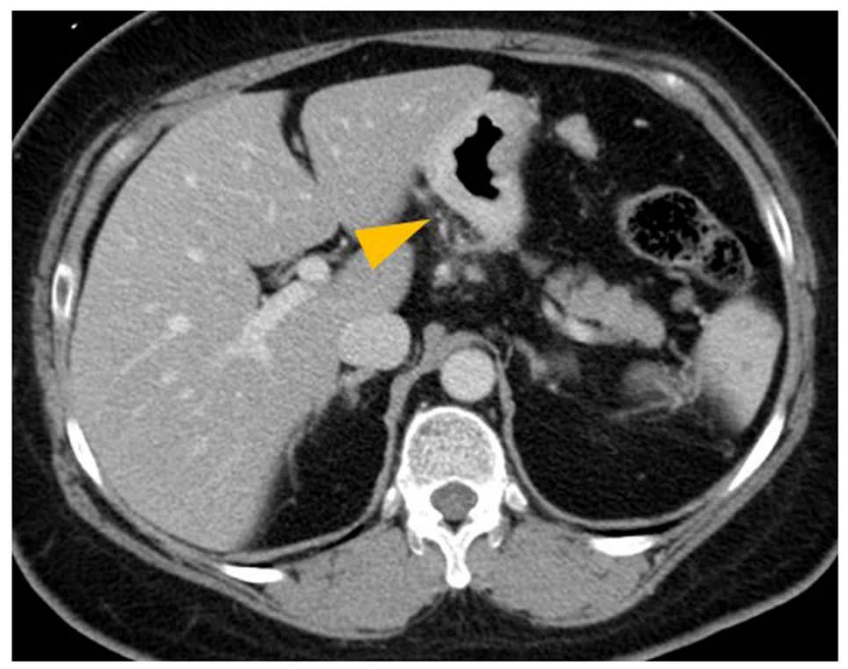

Figure 1. CT image showing thickening of the gastric body wall with contrast enhancement (arrow). CT, computed tomography.

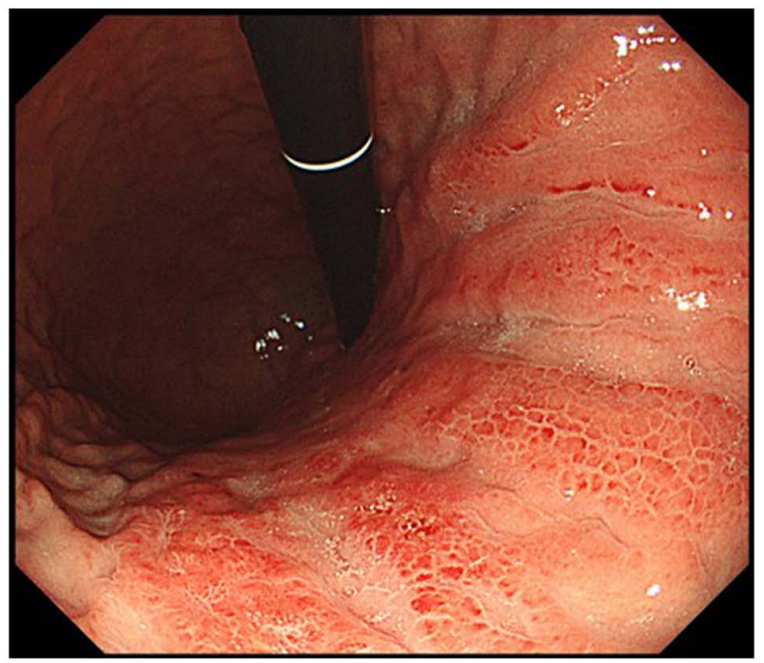

Figure 2. Endoscopic view. Fold thickening with erosion was evident on the lesser curvature of the upper to lower body.
A

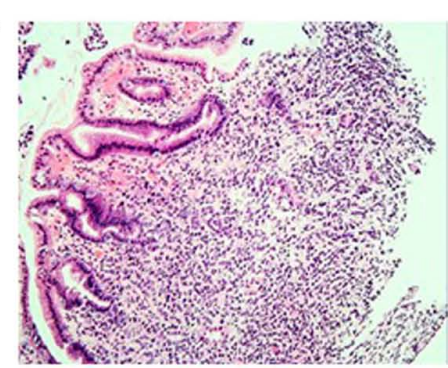

D

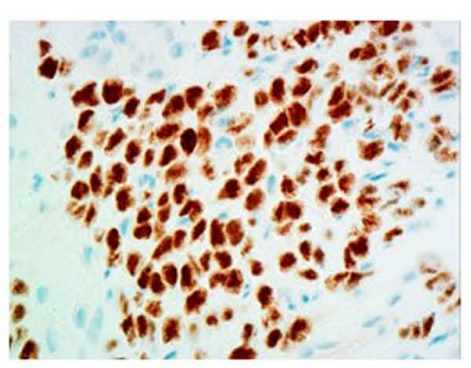

B

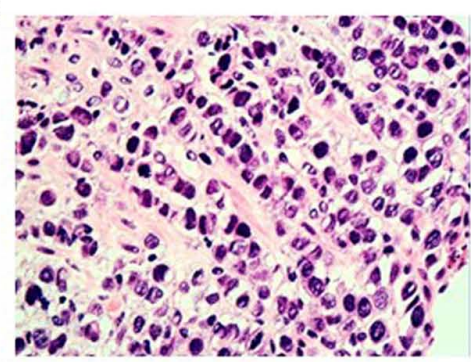

E

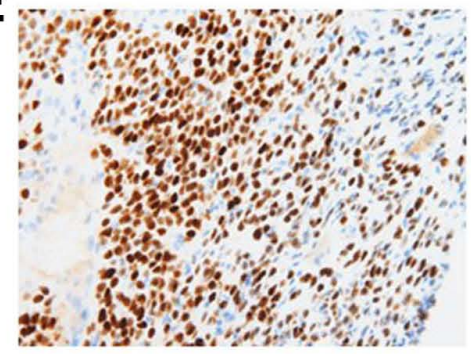

C

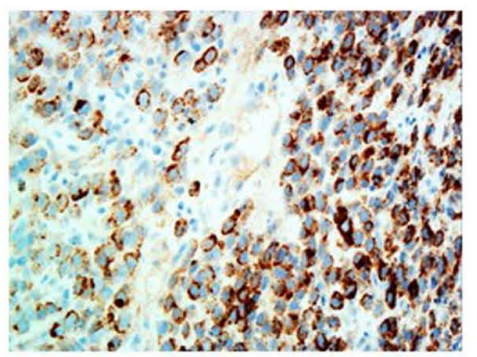

$\mathbf{F}$

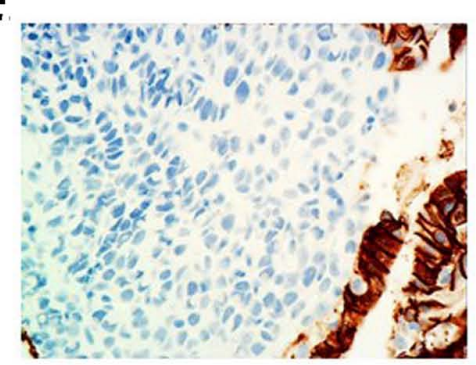

Figure 3. Gastric biopsy images showing infiltration of undifferentiated neoplasm with a single-file linear pattern within the gastric mucosa. (A) H\&E. Magnification, x40. (B) H\&E. Magnification, x400. Immunohistochemistry revealed positive staining for GCDFP-15, ER and PR. (C) GCDFP-15. Magnification, x400. (D) ER, Magnification, x400. (E) PR. Magnification, x400. Carcinoma cells were immunohistochemically negative for E-cadherin. (F) E-cadherin. Magnification, x400. ER, estrogen receptor; GCDFP-15, gross cystic disease fluid protein-15; H\&E, hematoxylin and eosin; PR, progesterone receptor.

fold thickening with erosion was observed on the lesser curvature of the upper to lower body on gastroscopy (Fig. 2). A biopsy specimen obtained from the gastric lesion contained lobular carcinoma, morphologically similar to signet ring cell carcinoma, as frequently seen in primary gastric cancer. Immunohistochemical examinations (Fig. 3) revealed positive staining for hormone receptors, mammaglobin and gross cystic disease fluid protein-15 (GCDFP-15), and negative staining for E-cadherin. These findings suggested that the gastric lesion represented a metastasis from the breast cancer. Positron emission tomography (PET)-CT showed no abnormal accumulations suggestive of metastasis in other organs. Palbociclib and fulvestrant were started for the gastric metastasis.
The patient provided written informed consent for her clinical data to be published in a medical journal.

\section{Discussion}

We have presented the case of a patient with ILC in whom gastric wall thickening was incidentally found on CT in the absence of symptoms, resulting in a diagnosis of gastric metastasis. This report emphasizes the importance of a clinical suspicion of gastric metastasis when abnormal image findings are detected for the stomach in patients with a history of ILC.

Gastric metastases from breast cancer are rare, accounting for 0.1 to $3.5 \%(8,9)$. Among invasive breast cancers, ILC is 
likely to metastasize to the gastrointestinal tract, including the stomach (12). ILCs are characterized by small, monomorphic, discohesive tumor cells with little nuclear atypia $(4,13,14)$. The growth pattern of ILC with a single cell infiltration pattern is due to the dysfunction of E-cadherin, a calcium-dependent transmembrane protein that controls cell-to-cell adhesion and suppresses tumor invasion and metastasis $(15,16)$. The lack E-cadherin expression is thus a hallmark of ILC.

Gastric involvement of metastatic breast cancer has been reported at a mean time of 6-7 years after breast cancer diagnosis (17). Most gastric metastases might thus be observed among metastases to multiple organs. The present case showed this first metastasis at 7 years after initial treatment. The mechanism of gastric metastasis from breast cancer with or without multiple organs remains unclear. The molecular analysis in many cases of gastric metastasis from breast cancer to clarify this issue should be awaited.

Gastric metastases are often asymptomatic due to diffuse spread under the mucosal layer, and are often found incidentally in advanced disease (18). Further, gastrointestinal symptoms such as indigestion, anorexia, dysphagia, and dyspepsia are non-specific for gastric metastases from breast cancer, and may be attributed to other metastases or side effects of other treatments such as chemotherapy $(10,19)$. Thus, the medical examination by interview may be unhelpful in arousing suspicion of gastric metastases. Indeed, our patient showed wide gastric metastasis that would easily have been overlooked without CT examination, due to the lack of any gastrointestinal symptoms.

Endoscopic features of gastric metastases are generally classified into two types: Those resembling submucosal tumor; and those resembling primary gastric cancer (20). Whereas a submucosal tumor-like lesion is the most frequent appearance of gastric metastases from all malignant tumors, gastric metastases from breast cancer mostly show diffuse infiltration similar to type 4 advanced gastric cancers $(10,19,21)$. A 'linitis plastica' appearance is characterized by the presence of diffuse infiltration to the submucosal and seromuscular layers of the stomach by tumor cells, which can cause fibrotic reaction leading to gastric wall thickening with reduced peristalsis (21). Radiologically, diffuse infiltration by tumor cells can be identified as gastric wall thickening on CT (22). In the present case, no abnormalities of the gastric mucosa were apparent on previous CT findings on retrospective comparison, indicating that CT might have captured the development of metastatic gastric cancer. In addition, PET-CT has been reported to offer limited sensitivity in detecting gastric tumors because of physiological ${ }^{18} \mathrm{~F}$-fluorodeoxyglucose (FDG) uptake and involuntary movements of the stomach (23). Furthermore, poorly differentiated types are known to show lower FDG uptake, which is likely affected by the low concentration of cancer cells, leading to high false-negative rates $(23,24)$. Concordant with these previous reports, no abnormal uptake was apparent on FDG-PET in the present case.

Gastric metastasis from ILC has no distinctive features in terms of cell morphology, invasive pattern, or endoscopic or radiologic features to facilitate differentiation from primary diffuse-type gastric cancer (25). In this respect, immunohistochemistry is the most important evaluation for differentiating gastric metastases (26). In the present case, the metastatic breast cancer showed positivity for ER, progesterone receptor (PR), and GCDFP-15 and negativity for E-cadherin, whereas primary gastric cancer is typically ER-, PR-, and GCDFP-15-negative and E-cadherin-positive (27,28). Expression of ER and PR is influential but not specific for the diagnosis of breast cancer metastasis. ER expression is relatively rare in primary gastric cancer. Previous reports have described finding ER and PR expressions in $10-30 \%$ of primary gastric cancers, but these expressions were generally focal or weak $(17,29)$. GCDFP-15 is known as an apocrine marker protein identified in apocrine glands, including those in the breast, sweat glands, and vulva (30). GCDFP-15 expression has been found in breast cancer with apocrine metaplasia, suggesting potential utility as a protein marker for breast cancer (31). A previous study reported that sensitivity reached up to $90 \%$ in ILCs with signet ring features, whereas only $55 \%$ of breast cancers showed positivity for GCDFP-15 (31). GCDFP-15 expression is also observed in other primary cancers such as those of the ovaries or lungs, but adenocarcinomas of the gastrointestinal tract are almost always negative for GCDFP-15 expression (32). Based on the results of immunohistochemical staining for these markers, we diagnosed gastric metastasis of breast cancer in this case.

In conclusion, consideration of gastric metastasis is important when incidental gastric findings are seen on imaging with or without symptoms in patients with ILC. Biopsies and immunohistochemical examination of the gastric lesion are necessary for accurate diagnosis and optimal treatment selection.

\section{Acknowledgements}

Not applicable.

\section{Funding}

No funding was received.

\section{Availability of data and materials}

All data generated or analyzed during this study are included in this published article.

\section{Authors' contributions}

YKa, YKo, and MO analyzed and interpreted the clinical patient data. YKo and MO drafted the manuscript. YKa performed the immunohistochemistry examination of the biopsy samples. KK formulated and designed the study. YD analysed and interpreted the histopathological data. MO critically revised the manuscript for important intellectual content. All authors read and approved the final manuscript.

\section{Ethics approval and consent to participate}

Not applicable.

\section{Patient consent for publication}

Written informed consent was obtained from the patient for the publication of this case report and the accompanying images. 


\section{Competing interests}

The authors declare that they have no competing interests.

\section{References}

1. Torre LA, Islami F, Siegel RL, Ward EM and Jemal A: Global cancer in women: Burden and trends. Cancer Epidemiol Biomarkers Prev 26: 444-457, 2017.

2. Arps DP, Healy P, Zhao L, Kleer CG and Pang JC: Invasive ductal carcinoma with lobular features: A comparison study to invasive ductal and invasive lobular carcinomas of the breast. Breast Cancer Res Treat 138: 719-726, 2013.

3. Siegel RL, Fedewa SA, Miller KD, Goding-Sauer A, Pinheiro PS, Martinez-Tyson D and Jemal A: Cancer statistics for Hispanics/Latinos, 2015. CA Cancer J Clin 65: 457-480, 2015

4. McCart Reed AE, Kutasovic JR, Lakhani SR and Simpson PT: Invasive lobular carcinoma of the breast: Morphology, biomarkers and 'omics. Breast Cancer Res 17: 12, 2015.

5. Ferlicot S, Vincent-Salomon A, Médioni J, Genin P, Rosty C, Sigal-Zafrani B, Fréneaux P, Jouve M, Thiery JP and Sastre-Garau X: Wide metastatic spreading in infiltrating lobular carcinoma of the breast. Eur J Cancer 40: 336-341, 2004.

6. Arpino G, Bardou VJ, Clark GM and Elledge RM: Infiltrating lobular carcinoma of the breast: Tumor characteristics and clinical outcome. Breast Cancer Res 6: R149-R156, 2004.

7. He H, Gonzalez A, Robinson E and Yang WT: Distant metastatic disease manifestations in infiltrating lobular carcinoma of the breast. AJR Am J Roentgenol 202: 1140-1148, 2014.

8. McLemore EC, Pockaj BA, Reynolds C, Gray RJ, Hernandez JL, Grant CS and Donohue JH: Breast cancer: Presentation and intervention in women with gastrointestinal metastasis and carcinomatosis. Ann Surg Oncol 12: 886-894, 2005.

9. Oda, Kondo H, Yamao T, Saito D, Ono H, Gotoda T, Yamaguchi H, Yoshida S and Shimoda T: Metastatic tumors to the stomach: Analysis of 54 patients diagnosed at endoscopy and 347 autopsy cases. Endoscopy 33: 507-510, 2001.

10. Taal BG, Peterse $\mathrm{H}$ and Boot $\mathrm{H}$ : Clinical presentation, endoscopic features, and treatment of gastric metastases from breast carcinoma. Cancer 89: 2214-2221, 2000.

11. Greene FL: Breast tumours. In: TNM classification of malignant tumours. Sobin LH, Gospodarowicz MK and Wittekind C (eds). 7th edition. Wiley-Blackwell, Oxford, pp181-193, 2009.

12. Korhonen T, Kuukasjärvi T, Huhtala H, Alarmo EL, Holli K, Kallioniemi A and Pylkkänen L: The impact of lobular and ductal breast cancer histology on the metastatic behavior and long term survival of breast cancer patients. Breast 22: 1119-1124, 2013.

13. Christgen $\mathrm{M}$ and Derksen P: Lobular breast cancer: Molecular basis, mouse and cellular models. Breast Cancer Res 17: 16, 2015.

14. de Groot JS, Ratze MA, van Amersfoort M, Eisemann T, Vlug EJ Niklaas MT, Chin SF, Caldas C, van Diest PJ, Jonkers J, et al: $\alpha \mathrm{E}$-catenin is a candidate tumor suppressor for the development of E-cadherin-expressing lobular-type breast cancer. J Pathol 245: 456-467, 2018.

15. Rakha EA, Patel A, Powe DG, Benhasouna A, Green AR, Lambros MB, Reis-Filho JS and Ellis IO: Clinical and biological significance of E-cadherin protein expression in invasive lobular carcinoma of the breast. Am J Surg Pathol 34: 1472-1479, 2010.

16. Berx G, Cleton-Jansen AM, Nollet F, de Leeuw WJ, van de Vijver M, Cornelisse C and van Roy F: E-cadherin is a tumour/invasion suppressor gene mutated in human lobular breast cancers. EMBO J 14: 6107-6115, 1995.
17. Schwarz RE, Klimstra DS and Turnbull AD: Metastatic breast cancer masquerading as gastrointestinal primary. Am J Gastroenterol 93: 111-114, 1998.

18. Ushida Y, Yoshimizu S, Horiuchi Y, Yoshio T, Ishiyama A, Hirasawa T, Tsuchida T and Fujisaki J: Clinicopathological features of metastatic gastric tumors originating from breast cancer: Analysis of eleven cases. World J Oncol 9: 104-109, 2018.

19. Pectasides D, Psyrri A, Pliarchopoulou K, Floros T, Papaxoinis G, Skondra M, Papatsibas G, Macheras A, Athanasas G, Arapantoni-Datioti P and Economopoulos T: Gastric metastases originating from breast cancer: Report of 8 cases and review of the literature. Anticancer Res 29: 4759-4763, 2009.

20. Kim GH, Ahn JY, Jung HY, Park YS, Kim MJ, Choi KD, Lee JH, Choi KS, Kim DH, Lim H, et al: Clinical and endoscopic features of metastatic tumors in the stomach. Gut Liver 9: 615-622, 2015.

21. D'Angelo F, Rampini A, Cardella S, Antolino L, Nigri G, Valabrega S, Aurello P and Ramacciato P: Breast cancer metastasis to the stomach. J Cancer Metastasis Treat 5: 30, 2019.

22. Insko EK, Levine MS, Birnbaum BA and Jacobs JE: Benign and malignant lesions of the stomach: Evaluation of CT criteria for differentiation. Radiology 228: 166-171, 2003.

23. Shimada H, Okazumi S, Koyama M and Murakami K: Japanese gastric cancer association task force for research promotion: Clinical utility of 18F-fluoro-2-deoxyglucose positron emission tomography in gastric cancer. A systematic review of the literature. Gastric Cancer 14: 13-21, 2011.

24. Yun M: Imaging of gastric cancer metabolism using 18 F-FDG PET/CT. J Gastric Cancer 14: 1-6, 2014.

25. Pera M, Riera E, Lopez R, Vinolas N, Romagosa C and Miquel R: Metastatic carcinoma of the breast resembling early gastric carcinoma. Mayo Clin Proc 76: 205-207, 2001.

26. Eo WK: Breast cancer metastasis to the stomach resembling early gastric cancer. Cancer Res Treat 40: 207-210, 2008.

27. O'Connell FP, Wang HH and Odze RD: Utility of immunohistochemistry in distinguishing primary adenocarcinomas from metastatic breast carcinomas in the gastrointestinal tract. Arch Pathol Lab Med 129: 338-347, 2005.

28. van Velthuysen ML, Taal BG, van der Hoeven JJ and Peterse JL: Expression of oestrogen receptor and loss of E-cadherin are diagnostic for gastric metastasis of breast carcinoma. Histopathology 46: 153-157, 2005.

29. Koike K, Kitahara K, Higaki M, Urata M, Yamazaki F and Noshiro H: Clinicopathological features of gastric metastasis from breast cancer in three cases. Breast Cancer 21: 629-634, 2014.

30. Viacava P, Naccarato AG and Bevilacqua G: Spectrum of GCDFP-15 expression in human fetal and adult normal tissues. Virchows Arch 432: 255-260, 1998.

31. Mazoujian G, Bodian C, Haagensen DE Jr and Haagensen CD: Expression of GCDFP-15 in breast carcinomas. Relationship to pathologic and clinical factors. Cancer 63: 2156-2161, 1989.

32. Gown AM, Fulton RS and Kandalaft PL: Markers of metastatic carcinoma of breast origin. Histopathology 68: 86-95, 2016.

This work is licensed under a Creative Commons Attribution-NonCommercial-NoDerivatives 4.0 International (CC BY-NC-ND 4.0) License. 